\title{
Materials Research in Reduced Gravity 2020
}

\author{
DOUGLAS M. MATSON $\mathbb{1 0},{ }^{1,8}$ ROBERT W. HYERS, ${ }^{2}$ \\ MICHAEL SANSOUCIE ${ }^{3}$ SHAUN MCFADDEN ${ }^{4}$ JONGHYUN LEE,${ }^{5}$ \\ WILHELMUS SILLEKENS, ${ }^{6}$ and TAKEHIKO ISHIKAWA ${ }^{7}$
}

\begin{abstract}
1.-Department of Mechanical Engineering, Tufts University, Medford, MA, USA. 2.-Mechanical and Industrial Engineering Department, University of Massachusetts, Amherst, MA, USA. 3.-NASA Marshall Space Flight Center, Huntsville, AL, USA. 4.-School of Computing, Engineering, and Intelligent Systems, Ulster University, Londonderry, UK. 5.-Department of Mechanical Engineering, Iowa State University, Ames, IA, USA. 6.-European Space Agency, ESA-ESTEC, Noordwijk, The Netherlands. 7.-Department of Interdisciplinary Space Science, JAXA, Tsukuba, Japan. 8.—e-mail: douglas.matson@tufts.edu
\end{abstract}

\section{INTRODUCTION}

Reducing gravitational effects such as thermal and solutal buoyancy enables investigation of a large range of different phenomena in materials science. Reduced gravity experiments can isolate phenomena otherwise obscured in ground-based experiments, leading to new discoveries that can improve materials and processes here on Earth. In addition to ground-based and short-term drop tower, reduced gravity aircraft or sounding rocket facilities, long-term experiments in microgravity have a long history-from the early days of spaceflight to current experiments on the International Space Station (ISS). The Materials Research in Reduced Gravity Symposium was conducted as part of the 149th TMS Annual Meeting \& Exhibition in San Diego, CA. It involved four sessions composed of 30 presentations with contributions from more than 12 countries. The sessions concentrated on three different categories of topics related to ongoing reduced gravity research: (1) programmatic and facility status, (2) solidification, and (3) thermophysical properties. The sessions concluded with a panel discussion on how to support upcoming activities related to the National Academies Decadal Survey. The symposium was sponsored by the TMS Extraction \& Processing Division, the TMS

Douglas Matson, Robert W. Hyers, Michael Sansoucie, Shaun McFadden, Jonghyun Lee, Wilhelmus Sillekens, and Takehiko Ishikawa are JOM Guest Editors for the topic Materials Research in Reduced Gravity.

(Received June 16, 2020; accepted July 5, 2020)
Materials Processing \& Manufacturing Division and the TMS Process Technology and Modeling Committee.

\section{A SOLIDIFICATION SNAPSHOT}

The papers in this topic represent a snapshot of some of the exciting new solidification processing activities currently underway on ground and in space to support reduced gravity experiments.

In "A Stable Dendritic Growth with Forced Convection: A Test of Theory using Enthalpy-based Modeling Methods," Andrew Kao et al. present an analytical study of how forced convection influences dendrite growth in undercooled pure nickel melts. They present an enthalpy-based technique coupled to a lattice Boltzmann method linked by convective thermal transport to obtain a simulation of anisotropic dendrite growth using a sharp interface model. In practice, this involves iterative evaluation using an adaptive cell size approach to demonstrate convergence. The results show promise in explaining how ground-based terrestrial rapid solidification experiments differ from space-based tests.

In "Tracking Evaporation during Levitation Processing of Nickel-based Superalloys on the ISS" by Jannatun Nawer et al., thermodynamic modeling of the activity of each element in solution is used to quantify evaporation in multi-component commercial nickel-based superalloys during ISS testing in microgravity. Tracking of evaporation impacts the accuracy and precision for thermophysical property measurements, ensures that differential loss of volatile species does not change the chemistry of the alloy during testing and ensures that astronaut health and safety mitigation procedures are in place to eliminate the potential for exceeding station dust exposure limits. 
Flow-induced anomalous nucleation during space testing is investigated in "Confirmation of Anomalous Nucleation in Zirconium" by Gwendolyn Bracker et al. The model explores how the shock waves associated with the collapse of flow-induced voids create localized regions of high pressure. Within these regions, the fluid becomes highly supercooled and the enhanced driving force leads to dynamic nucleation.

\section{MICROGRAVITY DECADAL SURVEY}

2020 represents a significant milestone in space research. For the past 20 years, there has been a continuous presence of humans living, working and conducting research in space aboard the International Space Station. NASA is in the process of critically evaluating how to prioritize activities over the next decade and is tasking the National Academies of Sciences, Engineering and Medicine to develop a comprehensive research strategy for the next decade of life and physical sciences research in space. Survey tasks are expected to include the review of current and emerging areas of space-related biological and physical sciences research, identifying the most compelling science priorities and outlining future facility and platform requirements, recommending approaches to development of a robust, resilient and balanced program of space research and assembling proof-of-concept research campaigns over a broad set of cost categories.

Readers are encouraged to support the activities of the National Academies during the ongoing factfinding mission by contributing to the upcoming
Decadal Survey 2023. NASA desires assistance in developing plans to meet the needs of exploration missions, provide concomitant terrestrial benefit and uniquely advance scientific knowledge. Solicitation of input from the greater scientific community is essential to make this effort a success. Research scientists and the general public are encouraged to submit white papers directly to the National Academies. These documents are anticipated to be stand-alone descriptions of critical activities, projects or professional topical studies that can support the NASA research mission in the coming decade. These papers will provide the basis for discussions by various Decadal Survey Committees as they assess how NASA's mission will evolve over the next 10 years and prioritize recommendations for future research opportunities.

For additional information about the white paper solicitation, readers are encouraged to contact the corresponding author, Douglas Matson, who currently serves as the 2020 President of the American Society for Gravitational and Space Research and as a member of the National Academies Committee on Biological and Physical Sciences in Space.

To read or download any of the papers in this special topic, follow the URL http://link.springer.c om/journal/11837/72/9/page/1 to the table of contents page for the September 2020 issue (vol. 72, no. 9) of JOM.

Publisher's Note Springer Nature remains neutral with regard to jurisdictional claims in published maps and institutional affiliations. 